\title{
Movilización de las narrativas y redes de apoyo en estudiantes con desempeño académico favorable: una revisión bibliográfica
}

Mobilization of narratives and support networks in students with favorable academic performance: a bibliographic review

\section{Mobilização de narrativas e redes de apoio em estudantes com desempenho acadêmico favorável: uma revisão bibliográfica}

Yudy Tatiana Barrera Cortes*, Daniela Agudelo Torres, \& Jenny Paola Burgos Díaz

Universidad de Boyacá

Citar este artículo así:

Barrera, Y. T., Agudelo, D., y Burgos, J. P. (2018). Movilización de las narrativas y redes de apoyo en estudiantes con desempeño académico favorable: una revisión bibliográfica. Revista Enfoques, 3(1), 107-127. http://dx.doi.org/

Derechos de autor: Licencia Creative Commons AtribuciónNoComercial-SinDerivadas 4.0 internacional y 2.5 Colombia (CC BYNC-ND 2.5 (O)

Recibido: julio 10 de 2017 Revisado: mayo 08 de 2018 Aceptado: mayo 16 de 2018

* Contacto: Yudy Tatiana Barrera Cortes, Psicóloga, Universidad de Boyacá, ytbarrera@uniboyaca.edu.co 
Resumen | El objetivo de este artículo es presentar una revisión sobre el desempeño académico y su relación con factores asociados. La atención se centra en la posibilidad de generar procesos de movilización, a partir de las narrativas de los estudiantes que tienen un bajo desempeño académico y desde la visibilización de los recursos posibilitadores de quienes tienen un desempeño académico favorable. La narrativa es entendida desde la perspectiva ontológica del ser, es decir, como organizadora de la realidad de las personas. A partir de la consultoría sistémica, se obtuvo un escenario de intervención que buscaba favorecer la emergencia de narrativas más centradas en alternativas de solución que en el problema. Para dar cuenta de esta revisión se presentan tres categorías: factores asociados, familia y consultoría, y construcción de escenarios. Estas categorías se organizaron a partir de la revisión de 50 artículos, cuya lectura permitió fortalecer la comprensión del fenómeno: elementos como la implicación de la familia o la posibilidad del lenguaje, en tanto escenario de intervención, se configuraron como ejes fundamentales dentro de la investigación.

Palabras Clave | desempeño académico, consultoría sistémica, narrativas, familia, factores asociados.

Abstract | The objective was to present a review of academic performance and its relationship with associated factors. The focus is on the possibility of generating mobilization processes from the narratives of students who have low academic performance, from the visibility of the enabling resources of those who have a favorable academic performance. The narrative was understood from the ontological perspective of being, that is, as the organizer of the reality of people. From the systemic consultancy, an intervention scenario was obtained that sought to favor the emergence of narratives more focused on alternative solutions than on the problem. To give an account of this review, three categories are presented: Associated factors, family and consulting and construction of scenarios, which were organized from the review of 50 articles that allowed to strengthen the understanding of the phenomenon where elements such as the involvement of the family or the possibility of language as an interventive scenario, are configured as fundamental axes in research.

Keywords | Academic performance, systemic consulting, narratives, family, associated factors. 
Resumo | o objetivo foi apresentar uma revisão do desempenho acadêmico e sua relação com fatores associados. O foco é sobre a possibilidade de gerar processos de mobilização das narrativas dos alunos com baixo rendimento escolar, uma vez que a visibilidade de facilitadores recursos que têm um desempenho acadêmico favorável. A narrativa foi entendida a partir da perspectiva ontológica do ser, isto é, como o organizador da realidade das pessoas. A partir da consultoria sistêmica, obteve-se um cenário de intervenção que buscou favorecer o surgimento de narrativas mais voltadas para soluções alternativas do que sobre o problema. Para dar conta desta revisão três categorias são apresentados: fatores associados, família e consultoria e construção de cenários, que foram organizados a partir da revisão de 50 artigos que permitiu uma maior compreensão do fenómeno em que elementos como o envolvimento da família ou A possibilidade da linguagem como cenário interventivo configurase como eixos fundamentais na pesquisa.

Palavras-chave | Desempenho acadêmico, consultoria sistêmica, narrativas, família, fatores associados.

\section{Introducción}

Este artículo presenta una revisión bibliográfica y teórica de las investigaciones recientes en torno a cómo se organiza el desempeño académico y cuáles son los diversos factores asociados. Esta perspectiva se instaura a partir del reconocimiento de los recursos posibilitadores, entendidos como las emergencias narrativas que surgen a partir de experiencias favorables de los estudiantes en su entorno académico. Es importante hacer énfasis en las implicaciones de lo narrativo, puesto que las experiencias desde el lenguaje dan cuenta de los procesos de comprensión y cambio de la realidad de los estudiantes. Como precisa Echeverría (2003): "El ser humano no es una forma de ser determinada, ni permanente. Es un espacio de posibilidad hacia su propia creación. $Y$ aquello que lo posibilita es precisamente la capacidad generativa del lenguaje" (p. 33).

En este sentido, el que los estudiantes comiencen a narrar sus experiencias con un tono apreciativo permitirá que se organicen alternativas para la movilización de sus recursos. Por el contrario, la emergencia de las limitaciones aparecerá en las narrativas de los estudiantes que estén más en sintonía con la dificultad, con evitar valorar su responsabilidad en su ejercicio académico, y con otorgarle un carácter externo a la responsabilidad de sus resultados, haciendo borrosa la posibilidad de cambio. Así mismo, podremos tener al estudiante que se narre configurando las prospectivas con el mundo a partir del déficit. 
Retomando la perspectiva de la Ontología del Lenguaje, de Echeverría (2003), podemos afirmar que la narración es el “(...) espacio de su propia creación”. El cambio está latente gracias a la capacidad de cambiar las narraciones. Es aquí donde surge la consultoría sistémica como escenario que favorece la transformación de los relatos a partir de diversos movimientos y elementos conversacionales, que buscan organizar las novedades narrativas de los estudiantes en prueba académica, para favorecer narraciones novedosas que configuran experiencias alternativas.

En este sentido es que se articula la necesidad de construir un escenario de movilización, a partir del cual van a ser posibles nuevas miradas sobre los alumnos que presentan dificultades en su proceso de aprendizaje. Con esto se logrará que el estudiante piense nuevas herramientas y genere hábitos de estudio que lo favorezcan. Este escenario es el que se reconoce como consultoría sistémica y que, según Gómez, Hincapié, Montoya, Moreno y Ramírez (2008), permite acciones de transformación y cambio. Estas acciones se trabajan a nivel individual, familiar y ecológico, por medio de procesos dialógicos reflexivos. De estas acciones emergen nuevas relaciones que se entretejen, construyendo redes conversacionales y redes sociales de apoyo; resignificando experiencias e historias de vidas, y estableciendo nuevas pautas relacionales donde otros mundos son posibles.

\section{Método}

La búsqueda de los artículos se realizó en páginas científicas como Redalyc, Scielo y ProQuest. Se buscaron palabras clave como consultoría sistémica, desempeño académico, familia, y narrativa conversacional. También se buscaron factores asociados relacionados al desempeño académico. Así, nos basamos en referencias empíricas y teóricas de un total de 50 artículos. A partir de un análisis en torno a elementos conceptuales y metodológicos de dichos artículos, estos fueron organizados en tres categorías: factores asociados, familia y consultoría sistémica.

Se obtuvieron las siguientes categorías de análisis para hacer un mejor análisis de la información y facilitar el proceso de redacción: factores asociados, familia, y consultoría sistémica y construcción de escenarios. 


\section{Factores asociados}

En esta categoría se encuentran factores como el social y el personal, entendiendo que existe una relación de estos con el comportamiento de los estudiantes y su desempeño académico. Así mismo, se menciona cómo se organizan los estudiantes en torno al ámbito académico, lo que pone en evidencia aspectos sociales y académicos.

\section{Familia}

Aquí se encuentran todos los aspectos familiares influyentes en el desempeño académico y cómo estos impactan el problema del estudiante. Pero también cómo estos se pueden transformar en un escenario posibilitador para el estudiante.

\section{Consultoría sistémica y construcción de escenarios}

Esta categoría se organiza a partir de experiencias de consultoría sistémica en diversos contextos, donde se hace énfasis en aspectos importantes como los diálogos generativos, la resignificación del problema a partir de la movilización de las narrativas, y el manejo de herramientas novedosas. Todo ello abre una mirada amplia que facilita la construcción de los guiones y las estrategias a utilizar durante la intervención.

\section{Resultados}

\section{Factores asociados}

En este apartado interesan todos los factores asociados que pueden estar teniendo impacto en el desempeño académico de los estudiantes. Inicialmente se contempla la investigación realizada por Rico y Gómez (2014) donde se indica que los profesores poseen un sistema de valores estructurado y complejo, que hace que la interacción facilite las transformaciones mutuas entre el estudiante y el profesor, generándose así un proceso de crecimiento y cambio. Por su parte, Guzmán y Pacheco (2014) establecieron que el desempeño académico de los estudiantes universitarios está determinado por un contexto en el que intervienen los docentes como orientadores y los estudiantes como constructores del conocimiento. 
Continuando con la exploración acerca de la importancia de los profesores frente al desempeño académico, encontramos que Montero, Villalobos y Valverde (2007) afirman que la edad de los profesores influye directamente el ámbito académico, pues quizá los profesores más jóvenes generan más empatía con sus alumnos. Del mismo modo, Covarrubias y Piña (2004) revelan que algunos profesores presentan una influencia positiva en el aprendizaje, la cual beneficia principalmente el desarrollo de las capacidades de pensamiento y académicas de los estudiantes.

Habría que resaltar la investigación de Garbanzo (2007) donde se explica que el grado de motivación académica y los problemas familiares y sociales influyen directamente sobre el desempeño académico. Complementando lo anterior, vemos que Torres y Rodríguez (2006) hallaron que los estudiantes que cuentan con el apoyo tanto económico como emocional de sus padres presentan un mejor rendimiento académico.

Investigaciones como las de Ferreyra (2007) reconocen que los alumnos de bajo rendimiento son los más afectados por factores que disminuyen el rendimiento, tales como: el género, la edad, el nivel de instrucción de los padres, el estar o no casado, el vivir con los padres, haber estudiado en entidades públicas, tener una madre activa en el mercado laboral. Por el contrario, Rodríguez, Fita y Torrado (2004) afirman que el género no es un factor diferenciador del rendimiento académico. Esta divergencia entre autores deja claro que hay diversidad en los resultados de las investigaciones.

Por otro lado, Soria-Barreto y Zúñiga-Jara (2014) y Gómez, Oviedo y Martínez (2011) aclararon que el género muestra una relación directa con el éxito académico, siendo las mujeres quienes ofrecen mejor respuesta a las demandas académicas y por consiguiente obtienen mejores resultados.

Es necesario recalcar la investigación de Vélez y Roa (2005) donde se identificaron factores más allá del contexto académico, como el consumo de alcohol que se presenta con mayor frecuencia entre los hombres. Por otra parte, reconocen que las relaciones de pareja influyen de manera tanto positiva como negativa en el desempeño académico, dado que el tener un apoyo emocional podría ser motivante para mejorar a nivel educativo. Por otro lado, Erazo-Santander (2012) concluyó que los estudiantes les piden poca colaboración a 
sus profesores, familiares o compañeros, dejando de lado factores positivos para mejorar el desempeño académico.

Para fortalecer el argumento de la investigación es importante aclarar que existe una gran diversidad de situaciones que pueden conllevar a un desempeño académico no favorable. En las investigaciones de Carvajal (2014) y Sancho (2012) se afirma que la institucionalidad, la familia, el ciclo vital en el que se encuentre el estudiante, el contexto en el que esté desarrollando sus actividades académicas y sociales, y su capacidad de adaptación son factores para tener en cuenta a la hora de analizar el desempeño. Al respecto, Giraldo (2007) afirma que la adaptación del individuo a su nuevo contexto universitario marca un punto trascendental para su desempeño en el ámbito académico.

Con respecto a las evaluaciones, Mamani (2015) afirmó que las principales causas para que el estudiante repruebe un examen y, por consiguiente, presente bajo desempeño académico son: la mala metodología del docente, las preguntas demasiado difíciles y la tendencia del profesor a calificar muy bajo. Por otro lado, Vargas, Ramírez, Cortes, Farfán y Heinze (2011) demostraron que las calificaciones que se obtienen en el ámbito de básica secundaria tienen una alta relevancia en la universidad, pues si fueron buenas en el ámbito escolar lo seguirán siendo en el universitario.

Dentro de este contexto, debe considerarse el estudio de Repáraz, Touron y Villanueva (1990), quienes hablan de cuatro rasgos influyentes en el ámbito universitario. Los estudiantes con un desempeño favorable se diferencian por ser más emotivos, reflexivos, defensivos y con mayor control de sí mismo; los alumnos con bajo desempeño parecen distinguirse por un menor control frente a situaciones estresantes.

Otros factores que presentan relación con el buen desempeño académico del estudiante son los personales como la motivación, la responsabilidad y la autonomía. Por ende, Izar, Ynzunza y López (2011) identifican a los estudiantes con mayor desempeño como aquellos comprometidos que no dudan en realizar un esfuerzo elevado en sus actividades académicas. Vale recalcar que estos estudiantes mantienen estas características en el nivel profesional y mantienen la proyección de adquirir más conocimientos. 
En relación con la autonomía del estudiante, Rivas et al. (2015) afirman que es fundamental la participación en y apropiación del estudiante de su proceso de aprendizaje ya que eso garantiza calidad en medio de la cantidad de estudiantes y/o profesionales con los cuales competirá en la vida laboral. Siguiendo la misma línea, Oliva y Narváez (2013) determinaron que la motivación y el interés por la asignatura es fundamental para obtener buenos resultados frente a las demandas de dicha materia; es esa motivación e interés lo que impulsa al estudiante a conseguir un buen desempeño académico.

\section{Familia}

En esta categoría presentamos las investigaciones relacionadas con el factor familia. Este factor es tratado con carácter diferenciado debido a la importancia que tiene, según se evidenció durante la revisión bibliográfica realizada. Resaltando la importancia de la familia, es relevante citar a Zuluaga (2007) quien concibe a la familia como la encargada de satisfacer las necesidades biológicas y afectivas de los individuos. De igual manera, es la familia la que responde por el desarrollo integral de sus miembros y por la adecuada inserción de estos en la cultura, esto es, la transmisión de valores para que se comporten como la sociedad espera de ellos. El núcleo familiar también ayuda a que se vaya construyendo la identidad individual, o sea, ciertas cualidades y características que permanecerán a través del tiempo.

Guevara y Paredes (2011) afirmaron que la familia es un factor determinante en el rendimiento académico del estudiante por la importancia que desempeña en su formación como individuo, debido a que es la familia el primer ente con el cual se socializa y el que va a estar presente a lo largo de la vida de cada persona.

En la misma línea, Zuluaga (2007) hace énfasis en la terapia familiar, que define como un cuerpo de teorías y técnicas que estudian al individuo en su contexto social. En ella se intenta modificar la estructura de la familia, ya que cuando se transforma dicha estructura se modifican consecuentemente las posiciones de sus miembros, especialmente, dentro de ese núcleo. Y, como resultado, se modifican las experiencias de cada individuo. Lo anterior se puede explicar desde el Principio de Totalidad que dice: "El todo es más importante que la suma de sus partes". Cada una de las partes está relacionada con el todo y al modificar solo una parte se provoca el cambio en todas ellas. Un ejemplo de ello es cuando se presentan 
dificultades en las pautas de crianza desde la infancia y el niño en su adolescencia genera conductas antisociales; debido a esto se ve afectado todo el sistema familiar.

Complementando la idea de la importancia de la familia, Jiménez (2008) resalta ciertos factores que no permiten el buen desempeño de los estudiantes en el aula de clase y que se caracterizan por estar relacionados con los padres: los conflictos familiares, el trabajo, y la falta de motivación y preparación de que adolecen algunos padres. Hoy día, la escasa conciliación entre la vida laboral y la familiar lleva a que muchos niños pasen buena parte del día no escolar viendo la televisión, jugando con sus amigos o haciendo sus tareas sin la supervisión de un adulto. Ello no favorece en nada los aprendizajes que el niño realiza diariamente. Es necesario entender que en los primeros años de vida familiar y escolar es donde mejor se puede manejar la plasticidad cerebral de los niños, y que lo adquirido en esos primeros años es lo que los va a acompañar a lo largo de la vida.

En relación con los acontecimientos familiares que llevan al buen desempeño académico, es pertinente citar a Ponce, Espericueta, Ramos, Cepeda y Villarreal (2010) quienes concluyen que el éxito es multidimensional, ya que está integrado por el éxito en la vida y en el desarrollo personal, familiar y profesional. También está vinculado con la dimensión familiar desde el rol de hijo(a), esposo(a) o padre (madre), y con algunos atributos valorables como amor, sinceridad, comunicación, tranquilidad, responsabilidad y educación.

Conviene distinguir el hallazgo realizado por Farooq, Chaudhry, Shafiq y Berhanu (2011) quienes hablan de la importancia del estrato socioeconómico de la familia. También hacen referencia al nivel de educación que tengan los padres, debido a que puede ser directamente proporcional con el desempeño académico del estudiante.

González (2015) investiga sobre los factores familiares significativos en el ámbito académico. Lo hace desde la perspectiva de los estudiantes, encontrando que estos consideran que el apoyo familiar es importante en todos los ámbitos, tanto para motivarlos como para elegir en libertad. Los jóvenes ven a sus padres como un ejemplo a seguir en su futuro y consideran que, al recibir su apoyo en concordancia con los intereses de cada cual, logran dar una perspectiva de futuro a sus vidas. 
Siguiendo la misma línea, es claro pensar que la familia del menor está vinculada, en gran medida, con el desempeño académico. En el estudio realizado por Rojas (2005) se encontró que las familias de los menores, que sufrían maltratos, estaban nuclearmente desfiguradas. En la mayoría de los casos estudiados, la madre cumplía un doble rol (mamá y papá). A la hora de indagar por el desempeño académico, los estudiantes no reconocían que se estuvieran presentando dificultades en el ámbito familiar; como tampoco lo reconocían los padres.

El estudio anterior continúa, poniendo en evidencia que manejar un lenguaje apropiado dentro de las relaciones familiares le facilita al estudiante mantener ese mismo lenguaje con sus compañeros de clase y cualquier persona con la que interactúe. Asimismo, asegura que el trato que se le brinde en el seno familiar influye en su desarrollo y en la adquisición de nuevos conocimientos en el aula de clase.

Como se ha denotado, la familia es un ente primordial en el desempeño académico del estudiante. Por ello, es necesario hacer un énfasis en la familia y su conexión con la enfermedad de este siglo, el estrés. Ello permite hablar de lo que sería llamado el estrés familiar. Al respecto, es importante retomar el estudio realizado por Mushtaq y Khan (2012), en el cual se concluye que el estrés familiar no tiene relación con el desempeño académico. Por el contrario, Dengra, Kalra y Malhotra (2013) establecen que sí genera influencia y que es mucho más significativa que factores como el sexo.

\section{Consultoría sistémica y construcción de escenarios}

Se presentan a continuación las investigaciones desarrolladas en torno a procesos de consultoría sistémica, enfatizando en la metodología, los resultados, y los cambios presentados en las poblaciones intervenidas. Estupiñán, Garzón, Niño y Rodríguez (2006) lograron definir la consultoría sistémica como una práctica reflexiva realizada en el escenario de los acontecimientos, que abre la posibilidad de transformación implícita en esta práctica investigativa y de intervención terapéutica.

Inicialmente se aborda la investigación de Ospina y Gallo (2011), quienes hacen referencia al cambio que presentan las familias y los profesionales que intervienen. El proceso permitió reconocer cómo los niños, niñas y jóvenes no son culpables de las dificultades que se presentan en la familia. De esta forma, se favorece el reconocimiento de la complejidad de

\section{$\boldsymbol{\epsilon} \mid 116$}


las relaciones y la necesidad de fortalecerlas para movilizar el cambio en la interacción, de manera que los implicados puedan dar cuenta de la experiencia subjetiva.

En esta línea, Gallo y Ospina (2010) realizaron una intervención individual con niños y jóvenes donde lograron reconocer cómo los aspectos negativos orientaban a los niños a permanecer en el problema. Esta comprensión se generó a partir de la externalización del problema, que permitió ver que el niño o joven no es el problema. De igual manera Pedraza, Perdomo y Hernández (2009) reconocieron que las familias construyen narrativas dominantes de los síntomas manifiestos del niño, mediante distinciones, prejuicios y creencias. Igualmente, las familias tienden a evidenciar coalición entre el niño y el cuidador, y culpabilización mutua entre los padres.

Continuando la exploración con Páez et al. (2012), vemos que los autores lograron comprender que un terapeuta, cuando hace referencia a sí mismo, logra poner en acción un recurso potente que le permite intensificar y exponer aspectos significativos de resistencia y fortaleza en su propia vida, posibilitando un encuentro generador de cambio para ambos, consultante y terapeuta. Se refieren al terapeuta debido a que también se convierte en un generador de cambios en el sistema de consulta, pues, como lo afirma Viloria (2012), el consultante no solo crea un cambio para sí. El consultante es definido, incluso, como un incentivador de acciones.

Al respecto conviene decir que, a partir de esos cambios que se generan en los dos ámbitos, tanto para el consultante como para el terapeuta, pueden surgir nuevas maneras de direccionar sus narrativas generando modelos, teorías, u otras perspectivas que nutren la posibilidad del cambio. A cerca de la teoría curricular, Giraldo (2007) y Leiva, Aristegui y Sanhueza (2014) establecen que efectivamente se pueden evidenciar cambios tangibles hacia visiones más humanas del mundo. Por su parte, de los trabajos de González (2006) y Biglia y Bonet (2009) se desprende un modelo de intervención, de consultoría sistémica, donde las historias vividas están necesariamente vinculadas a prácticas narrativas, pues contar las historias es la mejor manera de generar una resignificación del problema.

Por otro lado, Garzón (2008) aporta estrategias de intervención, consistentes en talleres diseñados para promover un aprendizaje creativo y motivante que permita al terapeuta la apropiación del conocimiento y el desarrollo de procesos autorreferenciales. También hace 
referencia al mapeo personal, el cual utiliza la bitácora de mapeo de historias personales y profesionales como un instrumento de desarrollo autorreferencial que le permite a cada terapeuta reconocer su estilo personal en la terapia y afinarlo, asociando su trabajo a los contextos sociales en los cuales se desarrolla.

En la misma línea, Castro (2013) indica la posibilidad de preguntas generativas que se pueden manejar en un escenario de consultoría sistémica para generar los diálogos generativos. Preguntas como: ¿Qué nuevas posibilidades tuvieron lugar? ¿Cómo contribuyen esas posibilidades a la comprensión y el desarrollo de la situación presente? ¿Qué posibilidades para nuevas acciones ofrece lo que ha ocurrido? ¿Cómo impactan las nuevas posibilidades en el problema actual? ¿Qué podrían significar a futuro? ¿Qué podría facilitar un cambio favorable en la relación entre ustedes? ¿Cómo podrían implementarse esos cambios? Este tipo de preguntas favorece procesos dialécticos reflexivos que constituyen una mediación útil para poder asumir la cibernética de segundo orden, en tanto dan cuenta de procesos autorreferenciales generativos que involucran los imaginarios mentales, conceptuales y paradigmáticos de los participantes y los investigadores.

Otra perspectiva hace referencia al estudio realizado por Jordán y Mariño (2007) en el cual se manejaron los escenarios de consultoría, a través del proceso de auto observación y meta observación, mediante conferencias virtuales y equipos conversacionales que permitieron co-construir nuevas interacciones entre los participantes, al motivarlos a narrar sus experiencias. Basándose en la construcción de identidad que transita por los diálogos, Linares (2010) muestra el impacto que genera el narrarse basado en el déficit, y evidencia la utilidad que tiene el proceso de intervención, para ampliar estos discursos deficitarios mediante la estrategia de involucrar las voces de otros, incluyendo los padres y los docentes de la institución educativa.

En la misma línea de argumentación, Márquez (2014) reconoce cómo los diálogos generativos mejoran la comunicación de todos los actores. Este tipo de diálogo también les permite sentirse valorados. A partir de este estudio, podemos hablar sobre los bucles reflexivos que, según Ballester y Colom (2012), son procesos transformadores, en los cuales es el investigador quien transforma el conocimiento, pero al mismo tiempo también se transforma a sí mismo. 
Cabe señalar el estudio realizado por Aya (2009), quien retoma los contextos relacionales haciendo énfasis en que en ellos se integran los procesos conversacionales, en los cuales circula la multiplicidad de textos y dificultades que organizan la experiencia de los seres humanos. Asimismo, se integran por procesos no conversacionales, los cuales refieren a interacciones que no incluyen diálogos explícitos, pero sí involucran una constante comunicación. La concepción de los procesos de construcción narrativa favorece la comprensión de los fenómenos de la vida humana, en tanto que permite interpretar varias dimensiones de los relatos.

Finalmente, es pertinente reconocer la caracterización de los escenarios de consultoría sistémica que, según Gómez, Hincapié, Montoya, Moreno y Ramírez (2008), permiten acciones de transformación y cambio trabajados a nivel individual, familiar y ecológico, por medio de procesos dialógicos reflexivos en donde emergen nuevas relaciones, que se entretejen construyendo redes conversacionales y redes sociales de apoyo, resignificando experiencias e historias de vidas, y estableciendo nuevas pautas relacionales donde otros mundos son posibles.

\section{Discusión}

El presente apartado da a conocer el análisis desarrollado a partir de la revisión bibliográfica presentada previamente. Se organiza en torno a las categorías manejadas durante el desarrollo de la revisión y se construye a partir de un panorama reflexivo y analítico sobre la necesidad de investigar, comprender e intervenir el fenómeno, dando cuenta de la consultoría sistémica como eje de la intervención.

\section{Factores asociados}

En esta categoría se encontró una diversidad de factores sociales y académicos que denotan la complejidad del fenómeno. En la actualidad, los estudiantes presentan dificultades debido a una cantidad suficiente de estímulos que influyen en cómo se desenvuelven los diferentes ámbitos. En este sentido, Guzmán y Pacheco (2014); Montero, Villalobos y Valverde (2007); Covarrubias y Piña (2004) coinciden al mencionar que influyen varios componentes relacionados con los docentes y la manera como se orientan las asignaturas.

Otro factor importante es el reconocimiento, por parte de los estudiantes, de las herramientas a que tienen acceso para mejorar sus procesos de aprendizaje, esto es, las tutorías 
brindadas por los profesores, las charlas y demás servicios disponibles, que los estudiantes no utilizan. En esto coincide Erazo-Santander (2012) quien concluyó que los estudiantes tienden a no solicitar apoyo de sus profesores, familiares o compañeros, y por este motivo se les dificulta resolver dificultades académicas y se les dificulta visibilizar posibles soluciones.

En esta misma línea, Guzmán y Pacheco (2014) concluyen que el desempeño académico de los estudiantes universitarios está determinado por un contexto en el que intervienen los docentes como orientadores y los estudiantes como constructores del conocimiento. Sin embargo, al parecer este rol no resulta lo suficientemente atractivo para los estudiantes, ya que en ocasiones no tienen intención de profundizar más allá de lo que se les brinda en la clase.

Finalmente, se reconoce que la metodología utilizada en las aulas de clase interviene en cómo el estudiante aprende y cómo logra interiorizar los conocimientos. Igualmente, importantes son la manera como el profesor interactúa con sus alumnos y el apoyo brindado por las tutorías con respecto a algún tema que no haya quedado claro, pues la construcción de conocimiento está en desarrollo constante y requiere de la participación de todos los actores involucrados.

\section{Familia}

Esta categoría permitió comprender la influencia de varios aspectos del entorno familiar, que hacen que el alumno responda a las demandas académicas. Los factores que cuentan son tanto las pautas de crianzas establecidas por los padres como en el tipo de apoyo emocional y monetario, así como también los valores, principios y creencias con que fueron educados en casa.

Inicialmente se observa que la comunicación y la forma de relacionarse entre padres e hijos constituyen un factor importante que influye en cómo pueda el estudiante lograr un desempeño académico favorable. Como lo menciona Rojas (2005), el lenguaje que se maneja dentro de las relaciones familiares tiene un impacto en cómo el estudiante construye relaciones en el contexto académico; de igual manera revela la importancia de los valores que ellos aprenden del contexto familiar. Su importancia radica en el establecimiento de una identidad que, se supone, es la identidad que el estudiante va a mantener durante toda su vida. 
De igual manera, Zuluaga (2007) manifiesta que los padres son los encargados de suplir las necesidades biológicas y afectivas a los individuos; la familia es el primer vínculo que tiene el ser humano y esto ayuda a que se relacione con la cultura donde construirá y pondrá en juego los valores y creencias que aprendió en casa. En esta relación el individuo crea su propia identidad, la que va consolidando a medida que va creciendo. Es a partir de la construcción de esa identidad que los individuos se permiten crear una narración de sí mismos, construyendo de esta manera su relación con la realidad, lo cual les va a permitir responder a las situaciones de la vida diaria, logrando tener una perspectiva de lo que son capaces de lograr.

Finalmente, respecto de esta categoría, cabe señalar que el sistema familiar actúa como punto de equilibrio para el ser humano, pues representa refugio, paz y apoyo. Sin embargo, no en todas las ocasiones ocurre de la misma manera. Por ende, la postura que tome el sistema familiar es la que va a permitirle al estudiante afrontar exitosamente las dificultades diarias o va a marcar su limitación ante la necesidad de enfrentar dichas dificultades.

\section{Consultoría sistémica y construcción de escenarios}

Es importante profundizar el concepto de consultoría sistémica de Estupiñán, Garzón, Niño y Rodríguez (2006). Estos autores la definen como una práctica reflexiva realizada en el escenario mismo de los acontecimientos, que permite generar cambios en el momento en que suceden los procesos conversacionales.

En este sentido, esta categoría permite evidenciar cómo, desde la consultoría sistémica y la diversidad de escenarios que crea esta metodología, se logra generar cambios en la población con que se trabaja. Particularmente, cómo los estudiantes que tienen un bajo desempeño académico se favorecen con alternativas para narrarse de manera distinta. De igual manera, esta perspectiva metodológica permite que los mismos investigadores movilicen una visión diversa y generativa de su propio problema académico.

Todo esto se logra a partir de los escenarios de consultoría sistémica, los cuales, según Gómez et al. (2008), permiten acciones de transformación y cambio que se trabajan a nivel individual, familiar y ecológico, por medio de procesos dialógico reflexivos en donde emergen nuevas relaciones, que se entretejen gracias a: la construcción de redes conversacionales y redes sociales de apoyo, la resignificación de experiencias e historias de vidas, y el establecimiento de nuevas pautas relacionales donde otros mundos son posibles. 
Finalmente, es importante reconocer que la metodología planteada favorece un ejercicio de investigación e intervención, siendo un elemento novedoso que permite comprender y movilizar los cambios en los participantes y los investigadores.

\section{Conclusiones}

La relación docente-estudiante es uno de los factores principales en el ámbito académico, debido a que los estudiantes se sienten más motivados, con una mejor actitud al asistir a clases y responder a las demandas que exige cada docente, cuando este logra crear un ambiente colaborativo de construcción de conocimiento.

El entorno familiar es el pilar del estudiante, ya que sus miembros se ayudan mutuamente a terminar de construir una identidad y a formar las habilidades para su proceso educativo. En la familia se generan interacciones de apoyo en cuanto a: el cumplimiento frente a demandas académicas, la orientación en la toma de decisiones, el cumplimiento de metas personales y profesionales, los estados de ánimo y la manera de afrontar las situaciones de la vida.

Es posible concluir que la motivación personal marca un punto trascendental en la adquisición de aprendizaje, ya que los estudiantes visualizan su futuro a partir del rol que desempeñan en el contexto universitario. Los estudiantes consideran que el tiempo dedicado al estudio, tanto fuera como dentro de la universidad, es indispensable para tener un desempeño favorable. Por tanto, el manejo que le dan a la organización de actividades curriculares y el tiempo libre es fundamental para los estudiantes que necesitan salir de prueba académica.

Aquí es importante reconocer la consultoría sistémica como la herramienta que va a generar diálogos reflexivos en los estudiantes, en donde el terapeuta se va a ver inmerso en el proceso de cambio, movilizando las narraciones negativas de los participantes hacia autorreferencias positivas sobre la situación de desempeño académico que están cursando. Esto les va a permitir evidenciar nuevos mundos y posibilidades para su realidad.

\section{Referencias}

Aya-Angarita, S. L. (2010). Reflexiones acerca de los procesos incluidos en la construcción narrativa. ¿Cómo emergen los relatos? Diversitas: perspectivas en psicología, 6(1), 185-194. Recuperado de http://www.scielo.org.co/pdf/dpp/v6n1/v6n1a15.pdf

$\boldsymbol{\epsilon} \| 122$ 
Ballester, L., y Colom, A. (2012). Intervención sistémica en familias y en organizaciones socioeducativas. Barcelona. España: Octaedro.

Biglia, B., y Bonet-Martí, J. (2009, January). La construcción de narrativas como método de investigación psico-social. Prácticas de escritura compartida. In Forum: Qualitative Social Research, 10(1), 1-24. Recuperado de https://ddd.uab.cat/pub/artpub/2009/146075/fqs_a2009v10n1.pd

Carvajal, A. (2016). Factores cualitativos que inciden en la deserción de la Educación Superior. Ponencia presentada en la IV Conferencia Congresos CLABES. Medellín, Colombia. Recuperado a partir de https:// revistas.utp.ac.pa/index.php/clabes/article/view/1050

Castro, N. (2013). Las interacciones comunicativas en los procesos de gestión de conocimiento en la universidad. Hacia un modelo de análisis. Question, 1(39), 16-28. Recuperado de http://sedici.unlp.edu.ar/bitstream/handle/10915/29613/Documento_completo.pdf?sequence=1

Covarrubias, P., y Piña, M. (2004). La interacción maestro-alumno y su relación con el aprendizaje. Revista Latinoamericana de Estudios Educativos (México), 34(1), 47-84. Recuperado de https://www.redalyc.org/ pdf/270/27034103.pdf

Dengra, M., Kalra, A., \& Malhotra, G. (2013). Study on factors affecting student quality of academic performance in colleges - with special reference to indore. Altius Shodh Journal of Management and Commerce, 2(1), 288-294.

Echeverría, R. (2003). Ontología del Lenguaje. Santiago de Chile, Chile: J.C. Sáez Editor.

Erazo-Santander, O. A. (2013). Caracterización psicológica del estudiante y su rendimiento académico. Revista Colombiana de Ciencias Sociales, 4(1), 23-41. Recuperado de https://dialnet.unirioja.es/descarga/articulo/5123816.pdf

Estupiñán, J., Garzón, D., Niño, J. A., y Rodríguez, L. (2006). Consultoría sistémica. Un enfoque interventivo, formativo, investigativo. Bogotá, Colombia: USTA.

Farooq, M. S., Chaudhry, A. H., Shafiq, M., \& Berhanu, G. (2011). Factors affecting students' quality of academic performance: a case of secondary school level. Journal of quality and technology management, 7(2), 1-14. Recuperado de http://pu.edu.pk/images/journal/iqtm/PDF-FILES/01-Factor.pdf 
Ferreyra, M. (2007). Determinantes del desempeño universitario: efectos heterogéneos en un modelo censurado. (Tesis de Maestría). Universidad Nacional de La Plata, La Plata, Argentina. Recuperado de http://www. depeco.econo.unlp.edu.ar/maestria/tesis/048-tesis-ferreyra.pdf.

Gallo, L., y Ospina, M. (2010). Intervención sistémica dirigida al cambio interaccional a partir de la transformación de relatos de identidad de niños/as y jóvenes de un centro de protección. (Tesis de Maestría). Pontificia Universidad Javeriana. Bogotá D. C., Colombia. Recuperado de https://repository.javeriana. edu.co/handle/10554/6318

Garbanzo, G. M. (2007). Factores asociados al rendimiento académico en estudiantes universitarios, una reflexión desde la calidad de la educación superior pública. Revista Educación, 31(1), 43-63. Recuperado de https://www.redalyc.org/pdf/440/44031103.pdf

Garzón, D. I. (2008). Autorreferencia y estilo terapéutico: su intersección en la formación de terapeutas sistémicos. Diversitas: Perspectivas en Psicología, 4(1), 159-171. Recuperado de http://www.scielo.org.co/ pdf/dpp/v4n1/v4n1a14.pdf

Giraldo, D. F. B. (2007). Currículo para situarse en el mundo en torno a la educación superior. Magistro, 1(2), 209222. Recuperado de https://dialnet.unirioja.es/descarga/articulo/4038502.pdf

Gómez, D., Hincapié, C., Montoya, M., Moreno, M., y Ramírez, C. (2008). Reflexiones y aportes de la consultoría en familia como estrategia para la construcción de relaciones democráticas en las familias (trabajo de pregrado). Universidad de la Salle, Bogotá, D. C., Colombia. Recuperado de http://repository.lasalle. edu.co/handle/10185/1736

Gómez, D., Oviedo, R., y Martínez, E. (2011). Factores que influyen en el rendimiento académico del estudiante universitario. TECNOCIENCIA Chihuahua, 5 (2), 90-97. Recuperado de https://www.redalyc.org/ html/310/31045571059/

González, A. (2015). Desempeño académico y relaciones familiares en la perspectiva de estudiantes de Psicología. Blog de docentes y estudiantes. Psicología UANL. Disponible en https:/ /psicologiauanl.wordpress. com/2015/09/21/desempeno-academico-y- relaciones-familiares-en-la-perspectiva-de-estudiantesde-psicologia/

\section{巨 $\| 124$}


González, J. (2006). Modelo de consultoría de los centros de educación superior para la intervención de la micro, pequeña y mediana empresa. Revista Digital Universitaria, 7(4), 1-15. http://www.revista.unam.mx/ vol.7/num4/art28/abr_art28.pdf

Guevara, E., y Paredes, L. (2011). La familia y otros factores influyentes en el rendimiento académico de los estudiantes de Psicología. Investigium IRE, 2(2), 133-145. Recuperado de http://investigiumire.iucesmag. edu.co/index.php/ire/article/view/25/24

Guzmán, R., y Pacheco, M. (2014). Comunicación familiar y desempeño académico en estudiantes universitarios. Zona Próxima, 20(1), 79-91. Recuperado de: http://www.redalyc.org/pdf/853/85331022008.pdf

Izar, J. M., Ynzunza, C. B., y López, H. (2011). Factores que afectan el desempeño académico de los estudiantes de nivel superior en Rioverde, San Luis Potosí, México. CPU-e, Revista de Investigación Educativa, 12. Recuperado de http://www.uv.mx/cpue/num12/opinion/Izar-desempeno academico.html

Jiménez, M. (2008). Influencia de los padres en el rendimiento escolar de sus hijos. México, D.F. México: Codice

Jordán, T., y Nariño, E. (2007). Redes conversacionales en la co-construcción de la Experiencia Familiar y su relación con el afrontamiento del lupus eritematoso sistémico (Trabajo de grado). Universidad de la Salle, Bogotá, Colombia. Recuperado de: repository.lasalle.edu.co/bitstream/10185/1761/1/T98.07\%20J635r. pdf

Leiva, J., Aristegui, R., y Sanhueza, J. (2014). Formación humana y transformación social: perspectiva dialógica en la educación de adultos para la acción social. En Congreso de la Asociación Latino Americana de Estudios. Congreso llevado a cabo en la ciudad de Chicago. DOI: 10.13140/2.1.1362.5604

Linares, E. (2010). La construcción de narrativas alternativas en el contexto escolar alrededor de los niños con diagnóstico de déficit de atención (Tesis de maestría). Universidad Javeriana, Bogotá, Colombia

Mamani, R. (2015). Factores que influyen en el bajo rendimiento académico en matemática I en los estudiantes de la carrera de ingeniería civil de la UAJMS2. Revista Ventana Científica. 6(10), 14-22

Márquez, L. (2014). Diálogos generativos y colaborativos como facilitadores de capacitación y de aprendizaje organizacional. Caso: Vendedores de Distribuciones TAT A PRECIO SAS (Tesis de Maestría). Universidad Nacional de Colombia, Bogotá, Colombia. 
Montero, E., Villalobos, J., y Valverde, A. (2007). Factores institucionales, pedagógicos, psicosociales y sociodemográficos asociados al rendimiento académico y a la repetición estudiantil en la Universidad de Costa Rica. Revista Electrónica de Investigación y Evaluación Educativa, 13 (02), 215-234. Recuperado de https://www.uv.es/RELIEVE/v13n2/RELIEVEv13n2_5.htm

Mushtaq, I., \& Khan. S. (2012). Factors Affecting Students' Academic Performance. Global Journal of Management and Business Research, 12(9), 17-22. Recuperado de https://globaljournals.org/GJMBR_Volume12/3-Factors-Affecting-Students-Academic.pdf

Oliva, P., y Narvaez, C. (2013). Percepción de rendimiento académico en estudiantes de Odontología. Educación Médica Superior, 27(1), 86-91. Recuperado de http://scielo.sld.cu/scielo.php?script=sci_ arttext\&pid=S0864-21412013000100011\&Ing=es\&tlng=es.

Ospina, M., y Gallo, L. (2011). Intervención sistémica: cambio de narrativas de identidad en un centro de atención infantil. Revista Latinoamericana de Ciencias Sociales, Niñez y Juventud, 2 (9), 827-846. Recuperado de http://www.scielo.org.co/pdf/rlcs/v9n2/v9n2a23.pdf

Pedraza, S., Perdomo, M., y Hernández, N. (2009). Terapia narrativa en la co-construcción de la experiencia y el afrontamiento familiar en torno a la impresión diagnóstica de TDAH. Universitas Psychologica, 8(1), 199-214. Recuperado a partir de http://revistas.javeriana.edu.co/index.php/revPsycho/article/ view $/ 10$

Páez, M., Cano, D., Giraldo, A., Giraldo, M., Pérez, Y., Sanín, H., y Zapata, J. (2012). Narrativas resilientes del terapeuta y generación del cambio terapéutico (trabajo de especialización). Universidad de Manizales, Manizales, Colombia.

Ponce, M., Espericueta, M., Ramos, E., Cepeda, M., y Villareal, N. (2010). El éxito en la dimensión familiar y valoral en Educación Superior: Repositorio Digital Institucional Instituto Politécnico Nacional. Recuperado de: http: //www.repositoriodigital. ipn.mx/handle/123456789/3829

Repáraz, C., Tourón, J., y Villanueva, C. (1990). Estudio de algunos factores relacionados con el rendimiento académico en 8o de EGB. Bordón, 42 (2), 167-178

Rico, D., y Gómez, M. (2014). La interacción de los valores entre profesor-estudiante: construcción y reconstrucción en el aula y en la relación formativa. Facultad de Psicología, Maestría en Psicología Educativa. Bogotá, Colombia: Universidad Católica de Colombia.

E 126 
Rivas, I. D., Díaz, G. M., Benítez, M. D. C., TresPalacios, M. D. C. R., Valdivia, O. J., y Artime, A. L. J. (2015). Rendimiento académico de estudiantes en asignaturas del área básica en policlínicos universitarios. $R e-$ vista Educación Médica del Centro, 7(1), 89-105. Recuperado de http://scielo.sld.cu/pdf/edu/v7n1/ edu08115.pdf

Rodríguez, S., Fita, S., y Torrado, M. (2004). El rendimiento académico en la transición secundaria-universidad. Revista de Educación, 334(1), 391-414. Recuperado de http://www.revistaeducacion.educacion.es/ re334/re334_22.pdf

Rojas, L. (2005). Influencia del entorno familiar en el rendimiento de niños y niñas con diagnóstico de maltrato de la escuela Calarcá de Ibagué (Monografía). Pontificia Universidad Javeriana. Ibagué, Colombia. Recuperado de http://www.javeriana.edu.co/biblos/tesis/medicina/tesis24.pdf

Sancho, B. (2012). La intervención con familias en el ámbito educativo: perspectiva sistémica (Trabajo de grado). Universidad de Valladolid. Escuela Universitaria de Educación. Palencia, España.

Soria-Barreto, K., y Zúñiga-Jara, S. (2014). Aspectos determinantes del éxito académico de estudiantes universitarios. Formación universitaria, 7(5), 41-50. https://dx.doi.org/10.4067/S0718-50062014000500006

Torres, L., y Rodríguez, N. (2006). Rendimiento académico y contexto familiar en estudiantes universitarios. Revista Enseñanza e Investigación en Psicología, 11(2), 255-270.

Vargas, I., Ramírez, C., Cortés, J., Farfán, A., y Heinze, G. (2011). Factores asociados al rendimiento académico en alumnos de la facultad de medicina: estudio de seguimiento a un año. Salud mental, 34(4), 301-308. Recuperado de http://www.scielo.org.mx/pdf/sm/v34n4/v34n4a2.pdf

Vélez, A., y Roa, C. (2005). Factores asociados al rendimiento académico en estudiantes de medicina. Educación médica, 8(2), 74-82. Recuperado de http://scielo.isciii.es/pdf/edu/v8n2/original1.pdf

Viloria, E. (2012). Dilemas de los psicólogos/terapeutas sistémicos en formación y la contribución de una intervención sistémica de apoyo en la construcción de su identidad (Tesis de Maestría). Pontificia Universidad Javeriana. Bogotá, Colombia.

Zuluaga, B. (2007, 9 de febrero). Una mirada de la familia desde la perspectiva sistémica. Relaciones familiares. [Mensaje en un blog]. Recuperado de https://beatrizzuluaga.wordpress.com/2007/02/09/las-relaciones-de-pareja-y-su-influencia-en-los-hijos/ 